Editorial

\title{
Endoscopic Ear Surgery: Paradigm Shift or Subordinate Role?
}

\author{
II Joon Moon ${ }^{1}$ id $\cdot$ Sung Huhn $\mathrm{Kim}^{2}$ (i) \\ ${ }^{1}$ Department of Otorhinolaryngology-Head and Neck Surgery, Samsung Medical Center, Sungkyunkwan University School of Medicine, Seoul; \\ ${ }^{2}$ Department of Otorhinolaryngology, Yonsei University College of Medicine, Seoul, Korea
}

Until recently, the microscopic approach to the middle/inner ear has been a mainstay of ear surgery. However, with the development of high-definition endoscopic systems and specifically designed instruments, using the endoscope in the field of otology has become more popular within last decade. Endoscopic ear surgery (EES), or transcanal endoscopic ear surgery (TEES), is defined as using a rigid endoscope instead of using a microscope for middle and inner ear surgery via the transcanal approach. TEES has become a hot topic as it has multiple advantages. Endoscopes allow surgeons to see better and more clearly, especially hidden spaces in the middle ear cavity, because the use of angled endoscopes, such as $30^{\circ}, 45^{\circ}$, or $70^{\circ}$, provides a wide field of view. Consequently, endoscopes reduce the likelihood that the postauricular approach will be required for wide exposure, thereby avoiding the associated morbidity, and allow surgeons to use the transcanal approach in treating most diseases confined to the middle ear. Moreover, additional mastoidectomy and posterior tympanotomy can be avoidable in certain cases, and functional ear surgery can be achievable.

The number of publications on this topic worldwide has exploded in recent years, and several papers regarding TEES have recently been published in Clinical and Experimental Otorhinolaryngology [1-4]. Choi et al. [1] compared endoscopic tympanoplasty to conventional microscopic tympanoplasty and reported good outcomes, including a similar graft success rate and audiologic outcomes, shorter operation time, and less pain, without external postoperative scars. A systematic review and meta-analysis of endoscopic tympanoplasty also indicated that EES is less invasive, and decreases the canaloplasty rate, wound complications, and operation time [4]. In addition, patients receiving TEES reported higher cosmetic satisfaction with a comparable graft success rate and hearing outcomes.

From the perspective of cholesteatoma surgery, better visualization with an endoscope can potentially reduce cholesteatoma recurrence rates. Park et al. [3] showed that congenital cholesteatoma could be successfully removed via the transcanal endoscopic approach in patients with Potsic stage I, II, and III. Although the follow-up period was not long enough, the recurrence rate was only $4 \%$, which is better or comparable to the recurrence rate from previous studies. Bae et al. [2] also reported the outcome of endoscopic attic cholesteatoma surgery, and there were no significant differences between the endoscopic and microscopic groups in terms of hearing improvement, recurrence rate, and operation time. The authors [2] concluded that the endoscopic approach for management of attic cholesteatoma is comparable to the microscopic approach.

As mentioned above, TEES seems to have meaningful advantages over classic ear surgery techniques. Using endoscopes, surgeons can complete more work via the transcanal approach and avoid unnecessary dissection, resulting in functional ear surgery such as functional endoscopic sinus surgery. Moreover, the use of endoscopes to access the lateral skull base opens the door to new surgical techniques [5]. However, there are some disadvantages ofTEES, such as the one-handed technique, a loss of depth perception, possibility of inner ear damage by heat, and the need for training. In addition, long-term follow-up results are needed to clarify the advantages of TEES over the conventional approach.

We still cannot anticipate future trends in ear surgery. However, with the increased development of technology, refinement of surgical approaches, and conduction of long-term prospective studies, EES will likely be incorporated into widespread practice in otology and a paradigm shift may occur in the near future.

\section{CONFLICT OF INTEREST}

No potential conflict of interest relevant to this article was reported.

Copyright (C) 2019 by Korean Society of Otorhinolaryngology-Head and Neck Surgery.

This is an open-access article distributed under the terms of the Creative Commons Attribution Non-Commercial License (http://creativecommons.org/licenses/by-nc/4.0)

which permits unrestricted non-commercial use, distribution, and reproduction in any medium, provided the original work is properly cited. 


\section{ORCID}

Il Joon Moon https://orcid.org/0000-0002-3613-0734

Sung Huhn Kim https://orcid.org/0000-0003-4408-3066

\section{REFERENCES}

1. Choi N, NohY, ParkW, Lee JJ, Yook S, Choi JE, et al. Comparison of endoscopic tympanoplasty to microscopic tympanoplasty. Clin Exp Otorhinolaryngol. 2017 Mar;10(1):44-9.

2. Bae MR, Kang WS, Chung JW. Comparison of the clinical results of attic cholesteatoma treatment: endoscopic versus microscopic ear surgery. Clin Exp Otorhinolaryngol. 2019 May;12(2):156-62.

3. Park JH, Ahn J, Moon IJ.Transcanal endoscopic ear surgery for congenital cholesteatoma. Clin Exp Otorhinolaryngol. 2018 Dec;11(4): 233-41.

4. Lee SY, Lee DY, Seo Y, Kim YH. Can endoscopic tympanoplasty be a good alternative to microscopic tympanoplasty? A systematic review and meta-analysis. Clin Exp Otorhinolaryngol. 2019 May; 12(2):145-55.

5. Marchioni D, Alicandri-Ciufelli M, Rubini A, Presutti L. Endoscopic transcanal corridors to the lateral skull base: initial experiences. Laryngoscope. 2015 Sep;125 Suppl 5:S1-13.

Received February 8, 2019 Accepted February 14, 2019 